\title{
The EUSO-SPB mission
}

\author{
Valentina Scotti* \\ INFN sezione Napoli \\ E-mail: scottiv@na.infn.it
}

\section{Giuseppe Osteria}

INFN sezione Napoli

\section{for the JEM-EUSO Collaboration ${ }^{\dagger}$}

EUSO-SPB (Extreme Universe Space Observatory - Super Pressure Balloon) is an experiment on board a super pressure balloon for a flight duration which may reach 100 days. The instrument was launched on the 25th April 2017 from Wanaka in New Zealand; the flight was terminated after 12 days aloft. The instrument is an updated version of the EUSO-Balloon one. It includes a full original JEM-EUSO PDM (Photon Detection Module with 2304 pixels), and an optical system with two Fresnel lenses with a side of 1 meter covering a field of view of nearly \pm 6 degrees. The main scientific objective is the first observation and measurements of Ultra High Energy Cosmic Ray Air Showers by looking down from near space with a fluorescence detector. The EUSO-SPB will also search for UV pulse like signatures from other objects as meteoroids, atmosphere TLEs, SQM, LSPs and it will measure slowly varying UV light as airglow, bioluminescence events over the ocean.

The European Physical Society Conference on High Energy Physics

5-12 July

Venice, Italy

* Speaker.

${ }^{\dagger}$ Full author list see http://jemeuso.riken.jp/en/members.html 


\section{The EUSO project}

EUSO (Extreme Universe Space Observatory) is a new type of observatory which observes transient luminous phenomena occurring in the Earth's atmosphere looking at them from space [1]. EUSO aims to study the most energetic component of the cosmic rays spectrum, the so called Extreme Energy Cosmic Rays (EECR), to do astronomy and astrophysics through the particles channel. The telescope will observe fluorescence and Cherenkov Ultra Violet (UV) photons generated by Extensive Air Showers (EAS) created by EECR. The observation from space has two advantages: the target volume is far greater than possible from the ground and it is possible to have full sky coverage.

The main objectives of the EUSO is to study physics and astrophysics from $\mathrm{E}>5 \times 10^{19} \mathrm{eV}$, focusing at $\mathrm{E} \sim 10^{20} \mathrm{eV}$ and above, to:

- identificate EECR sources by high-statistics arrival direction analysis;

- measure the energy spectra of individual sources (spectral shape, flux, power);

- understand and constrain acceleration and emission mechanisms.

The EUSO collaboration has developed several pathfinder missions (see Fig. 1) to calibrate the detector response, test its performance in air and space, raise the Technological Readiness Level of some of the components and improve our knowledge of the various detectors:

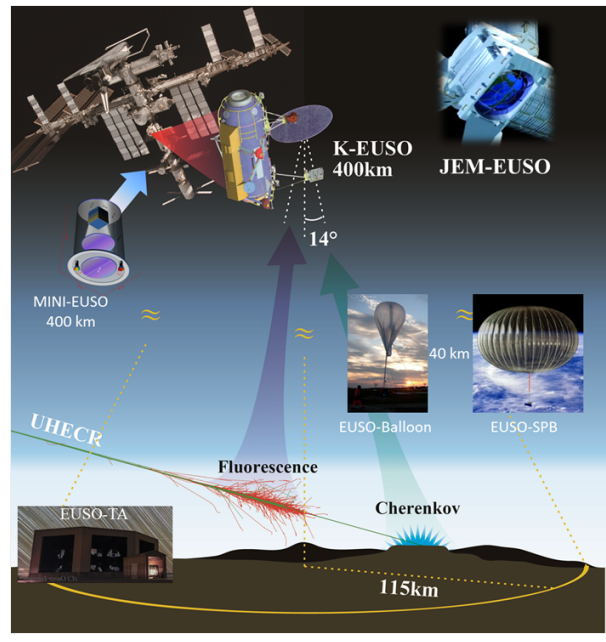

Figure 1: An overview of the EUSO missions.

- TA-EUSO, in 2014 a small telescope prototype was installed in the telescope array site in Utah (USA) to bring to maturity the technologies for EUSO. The telescope was composed by two Fresnel lenses of 1 meter of diameter and a Photo Detector Module (PDM) of 36 Multi-Anode PMT. This instrument is currently operational.

- EUSO-Balloon [3] a similar prototype was launched from Timmins (CA) on a stratospherical balloon by the French Space Agency in 2014. This was mainly a technology demonstrator, but it allowed the collaboration to perform trigger and background studies. 
- EUSO-SPB: the EUSO-Balloon instrument was launched for a long duration flight on a Super Pressure Balloon by NASA in April 2017. This mission will be described extensively in the following section.

- Mini-EUSO [2] a precursor on the International Space Station (ISS) approved by Italian and Russian Space agencies. It will be launched in early 2018 and it will bring one single EUSO PDM and two small Fresnel lenses (25 cm diameter) to ISS to perform UV background measurements. It is a key step for every mission in the UV range on ISS.

- K-EUSO foresees the installation of a bigger telescope on ISS in 2021-2022. This instrument will be equipped with Schmitd optics and could reach four times the exposure of the Pierre Auger Observatory.

\section{The EUSO-SPB mission}

The main goals of the EUSO-SPB mission were:

- make the first observations of Ultra High Energy Cosmic Ray Air Showers by looking down from suborbital space with an air fluorescence detector;

- measure UV background light at night from suborbital space.

In addition there were the search for faint and ultrafast UV signatures in the atmosphere from other phenomena and the establishment of methods and techniques for a future high energy astroparticle space observatory.

\subsection{The instrument}

The EUSO-SPB instrument was designed to operate at stratospheric altitude during nights with little or no moon, looking down to detect UV tracks from EASs. The working altitude was 33 kilometers and at that altitude the energy threshold for cosmic rays is $10^{18} \mathrm{eV}$. The instrument was composed by a refractive optical system incorporating two $1 \times 1 \mathrm{~m}^{2}$ Fresnel lenses with a BG-3 UV transmitting filter (330-400 nm), focusing the light in a $11.1^{\circ} \times 11.1^{\circ}$ field of view on concave focal surface on which is placed a Photo-Detector Module (PDM). The lenses are fabricated from UV transmitting polymethyl-methacrylate (PMMA). The $17 \mathrm{~cm} \times 17 \mathrm{~cm}$ PDM in the focus is composed of 36 Multi-Anode Photomultiplier Tubes (MAPMTs) containing 64 anodes, for a total of 2304 pixels. The PDM system also contains front-end ASICs, a first level trigger FPGA board, High Voltage (HV) and HV switches. The PDM is controlled by the Data Processing (DP). The signal coming from the PDM is digitized and processed by the data processor system which implements multiple trigger levels to filter out noise and identify events of interest. The data processor manages the storage of the data and the interfaces to the NASA science integration package (SIP) and telemetry. The DP consists of a 2nd-level trigger board, CPU board, Clock board, GPS board, house keeping board and low voltage power supplies [4].

The detector is completed by an infrared camera, to provide complementary information on the observation conditions, and a Silicon Photomultiplier Detector in a dedicated box. In addition, there are the NASA SIP, a solar power system, and a gondola exoskeleton frame to which all equipment was mounted including an antenna boom. 


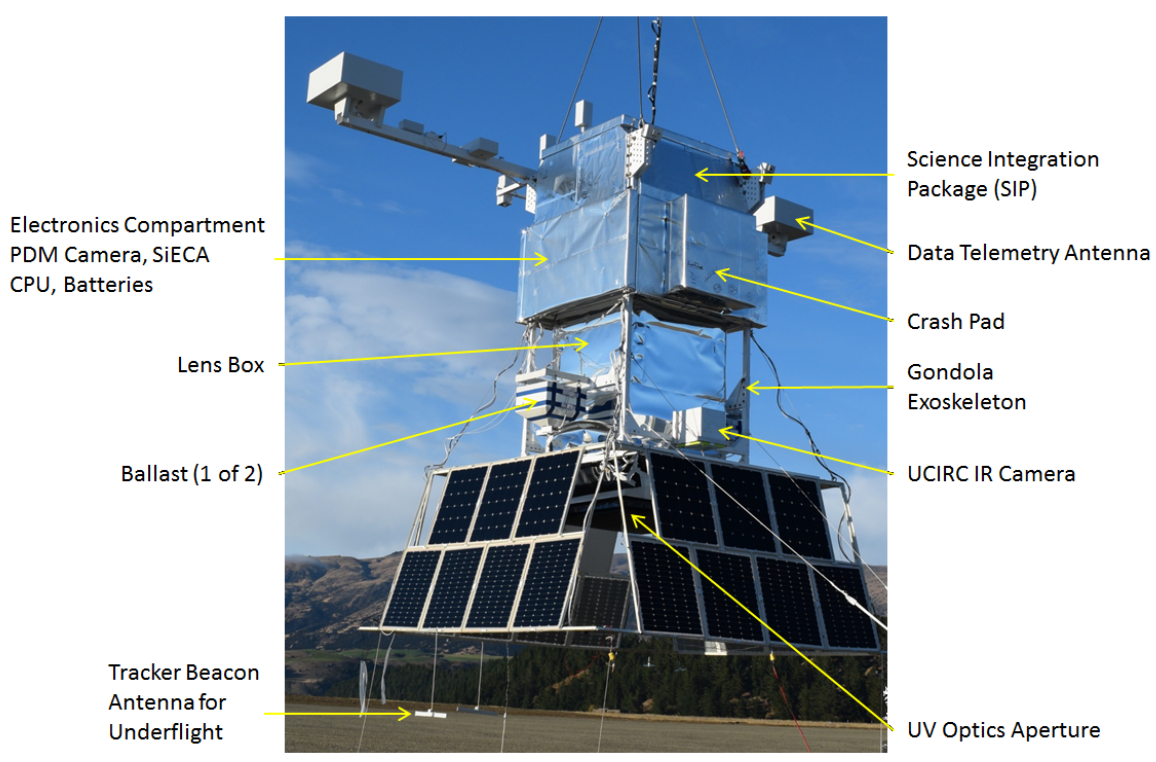

Figure 2: The EUSO-SPB1 instrument in flight ready configuration.

\subsection{The campaign}

The balloon mission was an effort of all the EUSO collaboration. After the Data Processor and PDM integrations in Europe, those systems were assembled with the optics coming from Japan in Colorado. The final step of this process were field tests in Utah Desert and hang tests in Texas at NASA center in Palestine. In December 2016 everything was shipped to new Zealand, where the flight campaign started in April 2017.

The launch occurred on April 24th 23:30 UTC from the Columbia Scientific Balloon Facility base in Wanaka. The launch was successful and the balloon reached the super pressure condition. After reaching the New Zealand coast, the balloon traveled northward passing about $30 \mathrm{~km}$ east of Christchurch before heading out over the Pacific ocean. Unfortunately the balloon developed an anomaly that lead to an early termination of the mission. 12 days after launch the entire flight train had to be "valved down"into the ocean about $300 \mathrm{~km}$ SE of Easter Island. In Figure 3 the altitude profile and the track of the balloon are shown.

A 24/7 shift schedule for EUSO-SPB1 instrument monitoring and operations was setup with 6 centers in Europe (France, Germany, Italy), RIKEN Japan, and Colorado US. A Cesna 421C aircraft to fly LEDs and a laser under the balloon after one orbit was in preparation [33]. During the flight 40 hours of PDM data were recorded over 11 nights. To facilitate downloading, data files were restarted automatically every 2 minutes. A shorter file was recorded at the start of each hour and downloaded with highest priority to provide detector monitoring. The data download bandwidth was reduced when 1 of the 2 links failed. 30 hours of the data recorded on board were downloaded.

Despite the setbacks, the EUSO-SPB1 instrument operated successfully while aloft and returned about $60 \mathrm{~GB}$ of data, taken in moon absence, which are now under analysis to search for UHECR and study the UV background, but also search for optical transients in progress and man- 


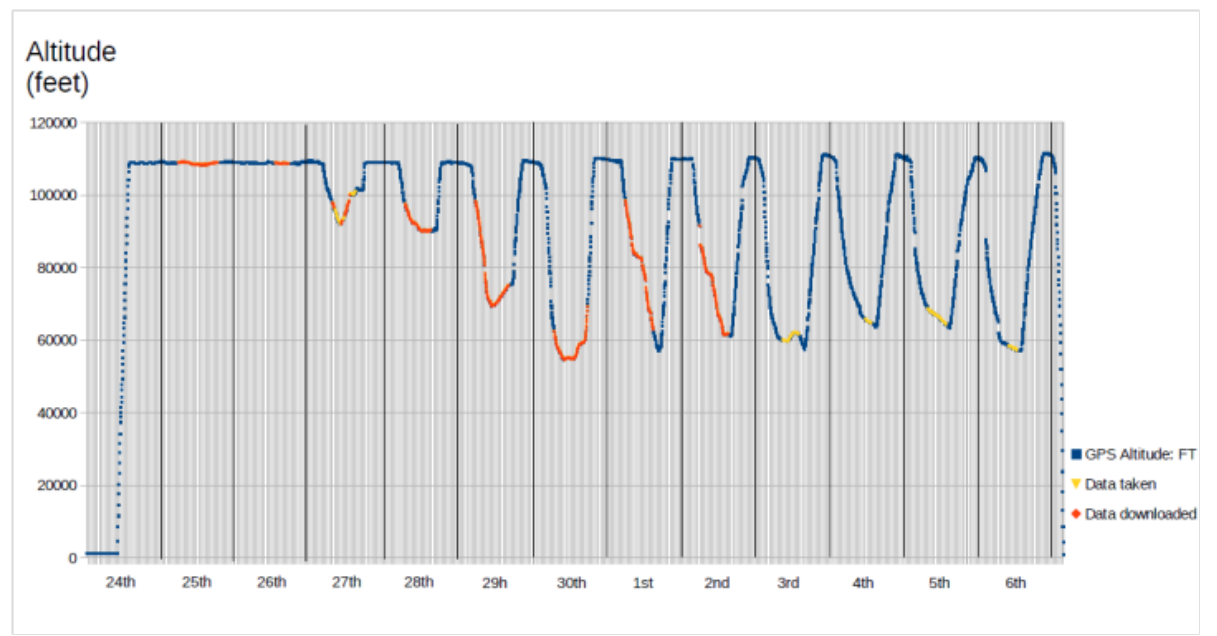

Figure 3: Altitude profile of the EUSO-SPB mission. The thicker lines denote the dark moonless periods when data was collected.

made flashes (planes, strobe lights).

\section{Conclusions}

An improved telescope prototype was built by the EUSO Collaboration and it met the NASA milestones. EUSO-SPB flew on a NASA super pressure balloon mission. The upgraded instrument worked properly during all the flight and a good amount of data was downloaded to ground. Data analysis and searches for UHECR are in progress.

Acknowledgment: This work was partially supported by NASA grants NNX13AH54G, NNX13AH52G, the French Space Agency (CNES), the Italian Space Agency through the ASI INFN agreement n. 2017-8-H.0, the Italian Ministry of Foreign Affairs and International Cooperation, the Basic Science Interdisciplinary Research Projects of RIKEN and JSPS KAKENHI Grant (22340063, 23340081, and 24244042), and the Deutsches Zentrum fur Luft und Raumfahrt, and the 'Helmholtz Alliance for Astroparticle Physics HAP'funded by the Initiative and Networking Fund of the Helmholtz Association (Germany). We acknowledge the NASA Balloon Program Office and the Columbia Scientific Balloon Facility and staff for extensive support, the Telescope Array Collaboration for the use of their facilities in Utah. We thank the Wanaka airport staff and manager Ralph Fegan. We also acknowledge the invaluable contributions of the administrative and technical staffs at our home institutions.

\section{References}

[1] JEM-EUSO Collaboration, Astropart.Phys. 44 (2013) 76-90, DOI: 10.1016/j.astropartphys.2013.01.008, arXiv:1305.2478 [astro-ph.HE]

[2] V. Scotti, G. Osteria, Nuclear Inst. and Methods in Physics Research A, (2016), doi:10.1016/j.nima.2016.06.042

[3] Scotti V. and Osteria G., on behalf of the JEM-EUSO Collaboration, Nuclear Inst. and Methods in Physics Research (2015), DOI: 10.1016/j.nima.2015.10.070

[4] V. Scotti, G. Osteria for the JEM-EUSO Collaboration,The Data Processor of the JEM-EUSO patfinders, doi: 10.1142/9789814603164_0019 